ARTICLE

\title{
Surface lattice engineering for fine-tuned spatial configuration of nanocrystals
}

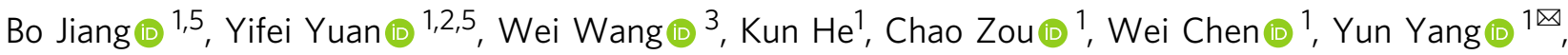 \\ Shun Wang (i) ${ }^{1 凶}$, Vitaliy Yurkiv (i) ${ }^{4 凶} \&$ Jun Lu (i) ${ }^{2 凶}$
}

Hybrid nanocrystals combining different properties together are important multifunctional materials that underpin further development in catalysis, energy storage, et al., and they are often constructed using heterogeneous seeded growth. Their spatial configuration (shape, composition, and dimension) is primarily determined by the heterogeneous deposition process which depends on the lattice mismatch between deposited material and seed. Precise control of nanocrystals spatial configuration is crucial to applications, but suffers from the limited tunability of lattice mismatch. Here, we demonstrate that surface lattice engineering can be used to break this bottleneck. Surface lattices of various Au nanocrystal seeds are fine-tuned using this strategy regardless of their shape, size, and crystalline structure, creating adjustable lattice mismatch for subsequent growth of other metals; hence, diverse hybrid nanocrystals with fine-tuned spatial configuration can be synthesized. This study may pave a general approach for rationally designing and constructing target nanocrystals including metal, semiconductor, and oxide.

\footnotetext{
${ }^{1}$ Nanomaterials and Chemistry Key Laboratory, Wenzhou University, Wenzhou, China. ${ }^{2}$ Chemical Sciences and Engineering Division, Argonne National Laboratory, Lemont, IL, USA. ${ }^{3}$ Department of chemistry and Center for pharmacy, University of Bergen, Bergen, Norway. ${ }^{4}$ Department of Mechanical and Industrial Engineering, University of Illinois at Chicago, Chicago, IL, USA. ${ }^{5}$ These authors contributed equally: Bo Jiang, Yifei Yuan. ${ }^{凶}$ email: bachier@163.com; shunwang@wzu.edu.cn; vyurkiv@uic.edu; junlu@anl.gov
} 
ybrid nanocrystals have various applications in many fields, including energy conversion ${ }^{1-3}$, catalysis ${ }^{4-10}$, cancer therapy ${ }^{11}$, and optics ${ }^{12-15}$. Accurate control over their spatial configuration (shape, composition, and dimension) is vital to adjusting their physicochemical properties, and consequently, to their effects on different functions ${ }^{16-20}$. Heterogeneous seeded growth, in which depositing one material on pre-made seeds composed of chemically different material, is the most widely used technology to construct these hybrid nanocrystals; the lattice mismatch of material pair strongly governs growth process and final spatial configuration ${ }^{21-26}$. Growth on a highly latticemismatched surface can increase system energy due to strain accumulation, and it thus prefers to grow only on one site, leading to low-symmetry Janus-like product with a small heterogeneous interface, for examples, $\mathrm{Cu}-\mathrm{Au}$ and $\mathrm{Cu}-\mathrm{Pd}$ material pairs ${ }^{27-32}$. In the case of slight lattice mismatch, homogeneous high-symmetry growth surrounding seeds is thermodynamically favored ${ }^{33-39}$, creating core-shell nanocrystals, for example, $\mathrm{Au}-\mathrm{Ag}$ material pair $^{34}$. Surely, achieving fine control over lattice mismatch can enhance our ability to tune nanocrystals spatial configuration. Currently, altering material pair is the only way to change lattice mismatch; yet, it is still impossible to fine tune lattice mismatch, since the lattice parameters of each material are fixed value and their difference is discontinuous. Consequently, the ability to precisely control the ultimate spatial configuration of nanocrystals is largely limited. Breaking this limitation will naturally make a path to unexplored construction of hybrid nanocrystals with fine-tuned spatial configuration.

In this work, we report a surface lattice engineering strategy, in which lattice mismatch is tuned by coating one material on seed surface instead of using different types of material pairs; moreover, the fine-tuning of lattice mismatch can be easily realized by controlling the amount of coated material, thus largely enhancing the ability to control the spatial configuration of nanocrystals. To prove that this approach has general applicability but not depends on shape, size, and crystalline structure of seeds, various Au nanocrystals including penta-fold twinned (PFT)

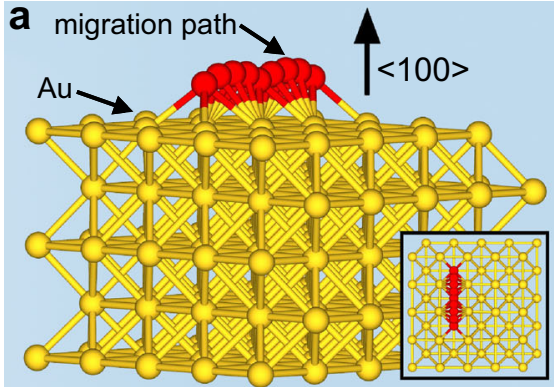

Au adatom migration on Pt-free $\mathrm{Au}(100)$

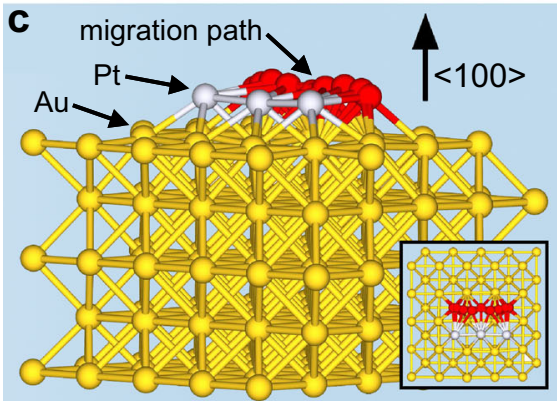

$\mathrm{Au}$ adatom migration on Pt-coated $\mathrm{Au}(100)$ nanodecahedrons (AuNDs), nanorods (AuNRs), nanobipyramids (AuNBs), and single crystalline nanoplates (AuNPs) and truncated octahedrons (AuTNOs) are chosen as seeds in this study, for their preparations can be realized using existing technologies ${ }^{40-46}$. Pt, which has a lattice mismatch larger than $3 \%$ relative to $\mathrm{Au}$, can largely influence the heterogeneous growth of $\mathrm{Au}^{47-50}$. Besides, hybrid nanocrystals composed of $\mathrm{Pt}$ and $\mathrm{Au}$ exhibit enhanced catalytic performance and multifunction owing to synergistic effects ${ }^{51-54}$, and thus we employed $\mathrm{Pt}$ as the lattice modifier. Different amount of Pt is coated on Au seeds surfaces, which generates adjustable lattice mismatch for subsequent growth of various metals ( $\mathrm{Au}, \mathrm{Ag}$, and $\mathrm{Pd}$ ) and consequently allows the formation of hybrid nanocrystals with fine-tuned spatial configuration. This approach is independent of the structure, shape and size of $\mathrm{Au}$ seeds. In addition, since lattice mismatch is universal in all crystal systems ${ }^{39}$, this strategy may pave a way toward the advancement of various hybrid nanocrystals with deterministically targeted spatial configuration.

\section{Results}

Density function theory (DFT) study. To evaluate the effect of Pt coating on subsequent growth of other metals, Au was taken as an example to perform DFT calculation. Similar to a common seed-mediated process, Au growth includes three stages: (1) formation of nucleation sites, (2) absorption of $\mathrm{Au}$ atoms on seed surface (the formation of $\mathrm{Au}$ adatoms), and (3) migration of adatoms to nucleation site to deposit ${ }^{36}$. The last stage essentially determines final spatial configuration of product. The Au nanocrystals (AuND, AuNR, AuNB, AuTNO) used in this work are bounded by $\{111\}$ and $\{100\}$ facets or stepped $\{100\}$ facets, which are energetically inactive surfaces with the energy of $\{100\}$ facet slightly higher than that of $\{111\}$ facet $^{55}$. Considering that $\mathrm{Pt}$ deposition preferentially occurs on high-energy positions, the effect of Pt on the migration of Au adatom on $\{100\}$ was investigated. Result shows that the activation energy ( $\left.E^{a c t}\right)$ of Au-on$\mathrm{Au}(100)$ migration is $0.5 \mathrm{eV}$ (Fig. 1a, b). When neighboring $\mathrm{Pt}$ atoms are present, the $E^{a c t}$ significantly decreases to $0.35 \mathrm{eV}$
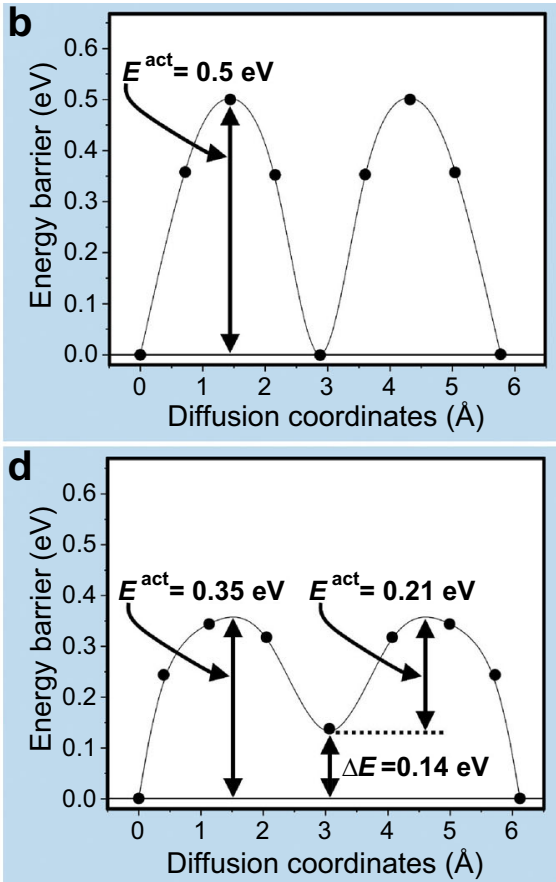

Fig. 1 DFT study and schematic adatom migration. a, b Au adatom migration on Pt-free $A u(100)$ surface, $\mathbf{c}, \mathbf{d}$ Au adatom migration on Pt-coated Au(100). The insets in $\mathbf{a}$ and $\mathbf{c}$ are top view of adsorbate structure. 

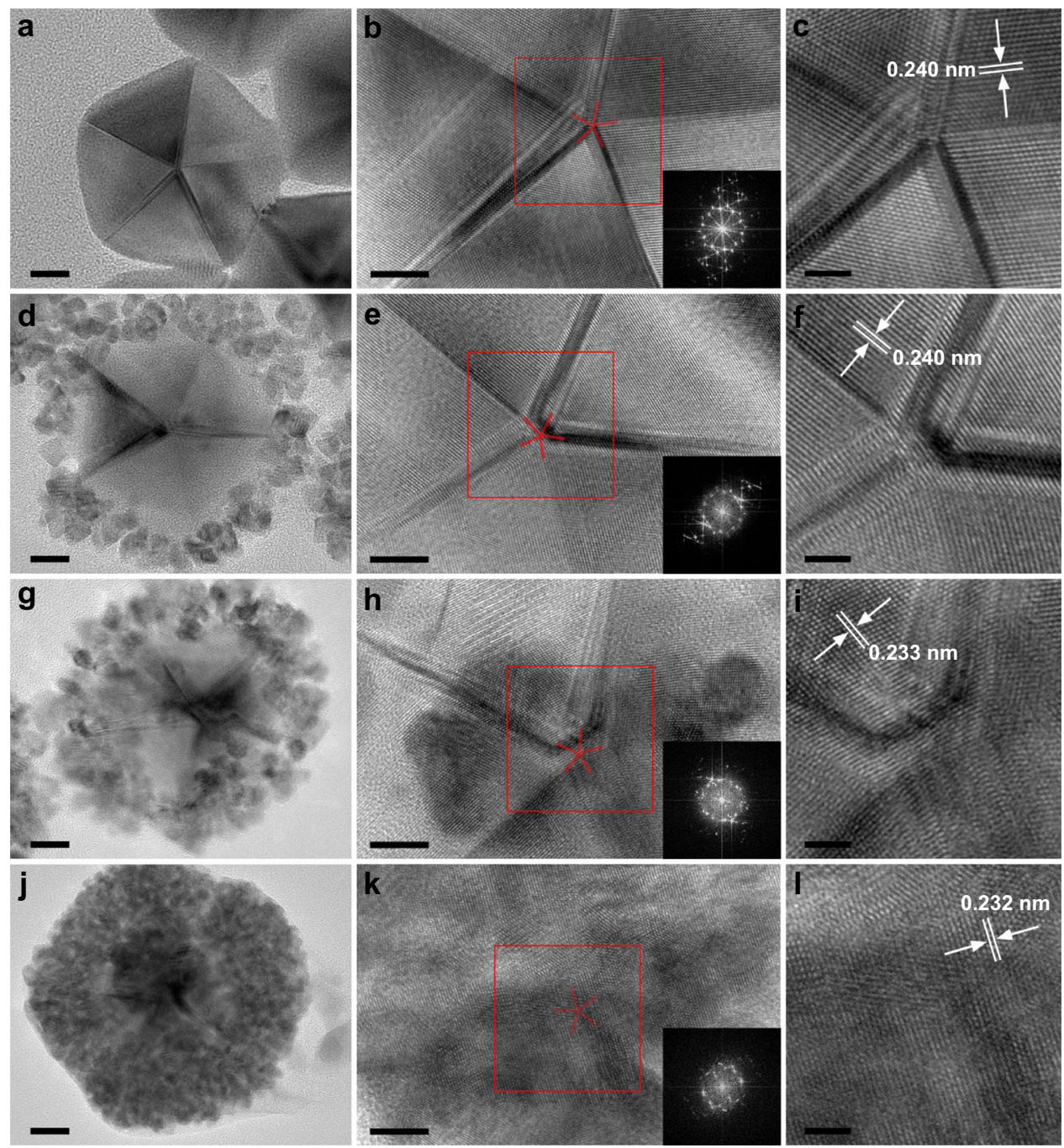

Fig. 2 Surface lattice engineering on AuNDs surface. HRTEM images of AuND@PtNC nanocrystals with different molar ratios of Pt and Au: a, b, c 0; d, e, $\mathbf{f} 0.5 ; \mathbf{g}, \mathbf{h}, \mathbf{i} 1 ; \mathbf{j}, \mathbf{k}, \mathbf{I}$ 2. The images in $\mathbf{c}, \mathbf{f}, \mathbf{i}, \mathbf{I}$ are the areas marked by the red boxes in $\mathbf{b}, \mathbf{e}, \mathbf{h}, \mathbf{k}$ and the insets are corresponding FFT patterns. Scale bars in $\mathbf{a}$, $\mathbf{d}, \mathbf{g}, \mathbf{j}$ are $10 \mathrm{~nm}$. Scale bars in $\mathbf{b}, \mathbf{e}, \mathbf{h}, \mathbf{k}$ are $5 \mathrm{~nm}$. Scale bars in $\mathbf{c}, \mathbf{f}, \mathbf{i}, \mathbf{I}$ are $2 \mathrm{~nm}$.

(Fig. 1c, d). Pt can increase Au diffusion coefficient by three orders of magnitude from $5.92 \times 10^{-15} \mathrm{~m}^{2} \mathrm{~s}^{-1}$ to $3.0 \times 10^{-12} \mathrm{~m}^{2} \mathrm{~s}^{-1}$, implying that the presence of $\mathrm{Pt}$ atoms leads to a high migration rate of $\mathrm{Au}$ adatoms. Wolf et al. draw a similar conclusion that negative strain (compressive) and positive strain (tensile) can speed up and slow down atomic diffusion, respectively ${ }^{56}$. Au $(4.078 \AA)$ has larger cell parameter than Pt $(3.924 \AA)^{57}$, so the migration of $\mathrm{Au}$ adatom on Pt-doped or Pt-coated $\{100\}$ should follow the negative strain pattern. Therefore, it is theoretically feasible to tune adatom migration and control growth pattern via surface lattice engineering.

Surface lattice engineering on AuNDs via Pt deposition. Inspired by the DFT results, surface lattice engineering was first applied to PFT AuNDs. High resolution transmitted electron microscopy (HRTEM) was used to investigate how surface lattice feature changes with Pt content (Fig. 2). For Pt-free AuNDs, PFT structure was clearly observed (Fig. 2a-c and Supplementary Fig. 2). HRTEM image shows a $0.240 \mathrm{~nm}$ inter-fringe distance which is attributed to the lattice spacing of $\mathrm{Au}(111) \mathrm{plane}^{58}$. After introducing small amount of $\mathrm{Pt}(\mathrm{Pt} / \mathrm{Au}=0.5: 1 \mathrm{~mol} / \mathrm{mol})$, Pt atoms were selectively deposited on the $\{100\}$ facets and adjacent $\{111\}$ facets of AuNDs to form Pt nanocircles (PtNCs) (Fig. 2d-f and Supplementary Figs. 1b, 3-5), producing AuND@PtNC nanocrystals ${ }^{59}$. PFT feature on most of $\{111\}$ facets was not strongly influenced. The lattice spacing of $\mathrm{Au}(111)$ plane near penta-fold axis has no changes, indicating that no Pt atoms were deposited here. However, the areas near five edges became rough because of preferential island-like deposition of Pt atoms. When the molar ratio of $\mathrm{Pt}$ and $\mathrm{Au}$ was increased to 1, surface crystalline feature significantly changed (Fig. 2g-i and Supplementary Figs. 1c, 6-8). Although the Pt growth on $\{100\}$ facet was still dominant, a considerable amount of $\mathrm{Pt}$ atoms were also deposited on $\{111\}$ facets, especially on the tip, as demonstrated by scanning transmitted electron microscopy (STEM) high angle annular dark field (HAADF) images and STEM energy dispersive X-ray spectroscopy (EDS) elemental maps. The lattice spacing of (111) plane, as a result of Pt doping, decreases to $0.233 \mathrm{~nm}$. The Pt deposition near the tip is closely related to reaction kinetics. When more Pt precursors are added into the reaction system, $\mathrm{Pt}$ atoms in solution increases, placing a high demand for nucleation sites. The surface atoms on the $\{100\}$ facets of AuNDs and adjacent positions were unable to provide enough nucleation sites, so nucleation occurred on other positions. The atoms on sharp tip are always active, which thus promotes Pt growth in these positions ${ }^{52}$. When the concentration of Pt precursor was 
further increased $(\mathrm{Pt} / \mathrm{Au}=2: 1 \mathrm{~mol} / \mathrm{mol}$ ) (Fig. $2 \mathrm{j}-\mathrm{l}$ and Supplementary Figs. 1d, 9), the fast growth kinetics of Pt forced almost all surface Au atoms of $\{111\}$ facets to act as nucleation sites and AuNDs were fully coated by Pt. Therefore, it is difficult to recognize the PFT structure using HRTEM. The lattice spacing of (111) plane is $0.232 \mathrm{~nm}$, which is smaller and closer to that of $\mathrm{Pt}(111)$ compared with former case. The fast Fourier transform patterns (FFT) also gave the same conclusion that Pt deposition makes the surface crystalline feature deviate from PFT structure. Clearly, surface crystalline feature of AuND can be engineered in two respects. (1) Controlled deviation from PFT structure. The crystalline feature change is attributed to the island-like deposition of small Pt particles, which formed many twinned boundaries and dislocations through ripening (Supplementary Fig. 7); therefore, the surface crystalline feature of AuND is deviated from PFT structure. (2) Fine tuning of lattice spacing. The lattice spacing of $\mathrm{Pt}(111)$ plane and $\mathrm{Au}(111)$ plane are about $0.230 \mathrm{~nm}$ and $0.240 \mathrm{~nm}$, respectively. The inter-diffusion of Pt coating and $\mathrm{Au}$ substrate results in formation $\mathrm{Pt} / \mathrm{Au}$ alloy ${ }^{36}$, especially on the AuND surface. Therefore, lattice spacing reduces with Pt content.

AuND-derived nanocrystals with controlled geometrical symmetry. Above results show that surface lattice engineering provides access to the fine tuning of crystalline structure and lattice spacing on AuND surface. For subsequent Au growths on these AuND@PtNC nanocrystals, their patterns should be related with Pt content because Pt-dependent lattice spacing brings adjustable lattice mismatch, which was confirmed by the following results. Pt-free AuNDs induced the formation of AuNBs which are almost symmetrical along penta-fold axis (Fig. $3 a-c$ and Supplementary Fig. 10a, b) ${ }^{58}$. When Pt-coated AuNDs (the Pt/Au molar ratio was 0.5 ) served as seeds, Au growth produced an interesting structure, in which each AuNB is coated by a PtNC (AuND@PtNC) (Fig. 3d-f and Supplementary Figs. 10e, f). The PtNCs are very useful markers for identifying the growth preference of $\mathrm{Au}$. The average lengths from the PtNC to one end are $150 \mathrm{~nm}$ and $90 \mathrm{~nm}$ to another end (Supplementary Fig. 11), suggesting that the growths along two directions are not equal; that is, the AuNB sections of AuND@PtNC nanocrystals are asymmetric along longitudinal direction. HRTEM images and FFT pattern show that the AuNB section in AuNB@PtNC has the same PFT structure as common AuNB (Supplementary Fig. 12h-m), suggesting that small amount of Pt has no effect on the crystalline feature of products. Once the molar ratio of $\mathrm{Pt}$ and $\mathrm{Au}$ in seeds was increased to 1 , almost half of products are still AuNB@PtNC nanocrystals, but Au growths created irregular Au domains in the rest (Fig. $3 g-k$ and Supplementary Fig. 10i, j). Both types of nanocrystals have lower longitudinal symmetry than the AuNB@PtNC shown in Fig. 3e; for example in AuNB@PtNC nanocrystals, the average length from the PtNC to one end increased to $160 \mathrm{~nm}$ and to another end decreased to $70 \mathrm{~nm}$ (Supplementary Fig. 13). Distinctly, high Pt content can further increase the difference of growth rate along two directions; high $\mathrm{Pt}$ content also caused large deviation of crystalline feature from PFT on seeds surfaces (Supplementary Figs. 7, 14), and thereby AuNDs lost their guiding effect, producing irregular $\mathrm{Au}$ domains. If the molar ratio of $\mathrm{Pt}$ and $\mathrm{Au}$ in seeds was 2, unidirectional growth occurred and yielded Janus-like (AuND@PtNC)-Au nanocrystals (Fig. 31-n and Supplementary Figs. $10 \mathrm{~m}, \mathrm{n}, 15,16)$, indicating that growth symmetry was further decreased. Meanwhile, almost all seeds surfaces lost PFT crystalline feature due to thick and dense Pt layer, which made each formed $\mathrm{Au}$ domain become irregular. The above results clearly demonstrate that nanocrystals spatial configuration can be controlled via this surface lattice engineering (Fig. 3o).
Growth mechanism. As discussed previously, the seeded growth process generally includes the formation of nucleation sites on seed surface, atom adsorption, and adatom migration to nucleation site and growth ${ }^{36}$. In our experiments, the formation of asymmetrical AuNB@PtNC nanocrystals demonstrates that preferential migration to one side occurred. Currently, it remains a grand challenge to in situ observe how Pt influences the product's asymmetry. Here, a mechanism is provided to explain the Ptdependent growth by using the AuNB@PtNC nanocrystal as an example.

First, one could reasonably hypothesize that the first nucleation site appearing on the surface of $\mathrm{Au}$ seeds may be randomly distributed on either side of the crystal but not in the Pt-coated edge region since the higher degree of lattice mismatch in the $\mathrm{Pt}$ coated region can prevent any $\mathrm{Au}$ nucleation ${ }^{60}$. As shown in Fig. 4, when a higher concentration of $\mathrm{Au}$ atoms create the firstly emerging nucleation site on one side of the AuND (supposed to be the right side as illustrated in the schematic), other $\mathrm{Au}$ adatoms would prefer to migrate to this active position to supply the growth of $\mathrm{Au}$ on this side because the nucleation site rich in atomic steps and defects is very active ${ }^{61}$. With the increase of synthesis time, the initially symmetric morphology of the $\mathrm{Au}$ seeds is gradually broken along longitudinal axis.

Second, it is certain that Pt coating can further increase the degree of asymmetry by facilitating the migration of Au adatoms from these Pt-coated surfaces to the nucleation sites to boost the growth of Au. For the used AuND nanocrystals, their five identical edges, in practice, are small-area $\{100\}$ facets but not lines in theory (Supplementary Fig. 2), indicating the selective coating of Pt on $\{100\}$ facets. As demonstrated by the DFT calculations (Fig. 1), the energy barrier for Au adatom diffusion on $\mathrm{Au}-(100)$ facets decreases when $\mathrm{Pt}$ is present, that is, Pt can speed up Au adatom diffusion by lowering the energy barrier of migration pathway. A faster migration rate allows more $\mathrm{Au}$ adatoms to migrate to the initially nucleated sites at a given time, which thus increases the degree of morphological asymmetry. This theory is further corroborated by the observation that a higher Pt content on AuNDs leads to an even higher degree of asymmetry as shown in Fig. 3. For the situation that $\mathrm{Au}$ nanocrystalline seeds are fully coated by thick Pt layer, Au growth can be directly considered to be on Pt surface; that is, the whole surfaces of seeds are highly lattice-mismatched to $\mathrm{Au}$ adatoms, indicating that $\mathrm{Au}$ adatoms on the surface should have a stronger trend (higher rate) to migrate to thermodynamically favorable position. Therefore, once a nucleation site is formed on either side of the seed, all $\mathrm{Au}$ adatoms from the other positions quickly migrate to the nucleated position due to its higher activity and smaller lattice mismatch, further causing selective growth only on that specific side ${ }^{56}$.

AuNR- and AuNB-based nanocrystals with controlled geometrical symmetry. General applicability of an approach is significant in nanocrystal synthesis. Here, one-dimensional PFT AuNRs coated by different amount of Pt were also used as seeds to verify the universality of surface lattice engineering; similar to the growth on AuND@PtNCs, product spatial symmetry is very sensitive to Pt content. Pt-free AuNRs induced the formation of round-tip AuNBs (Fig. 5a-c, and Supplementary Figs. 18, 19), and their lengths along transverse and longitudinal axis are larger than that of AuNR seeds. Therefore, it can be known that the growth occurred towards both directions, but the growth rate along the latter was faster; furthermore, they are geometrically symmetrical in each direction. When AuNR@Pt nanocrystals with 0.15 or 0.3 molar ratio of $\mathrm{Pt} / \mathrm{Au}$ were used as seeds (Fig. $5 \mathrm{~d}-\mathrm{i}$ and Supplementary Figs. 21-26), Au growth along transverse 


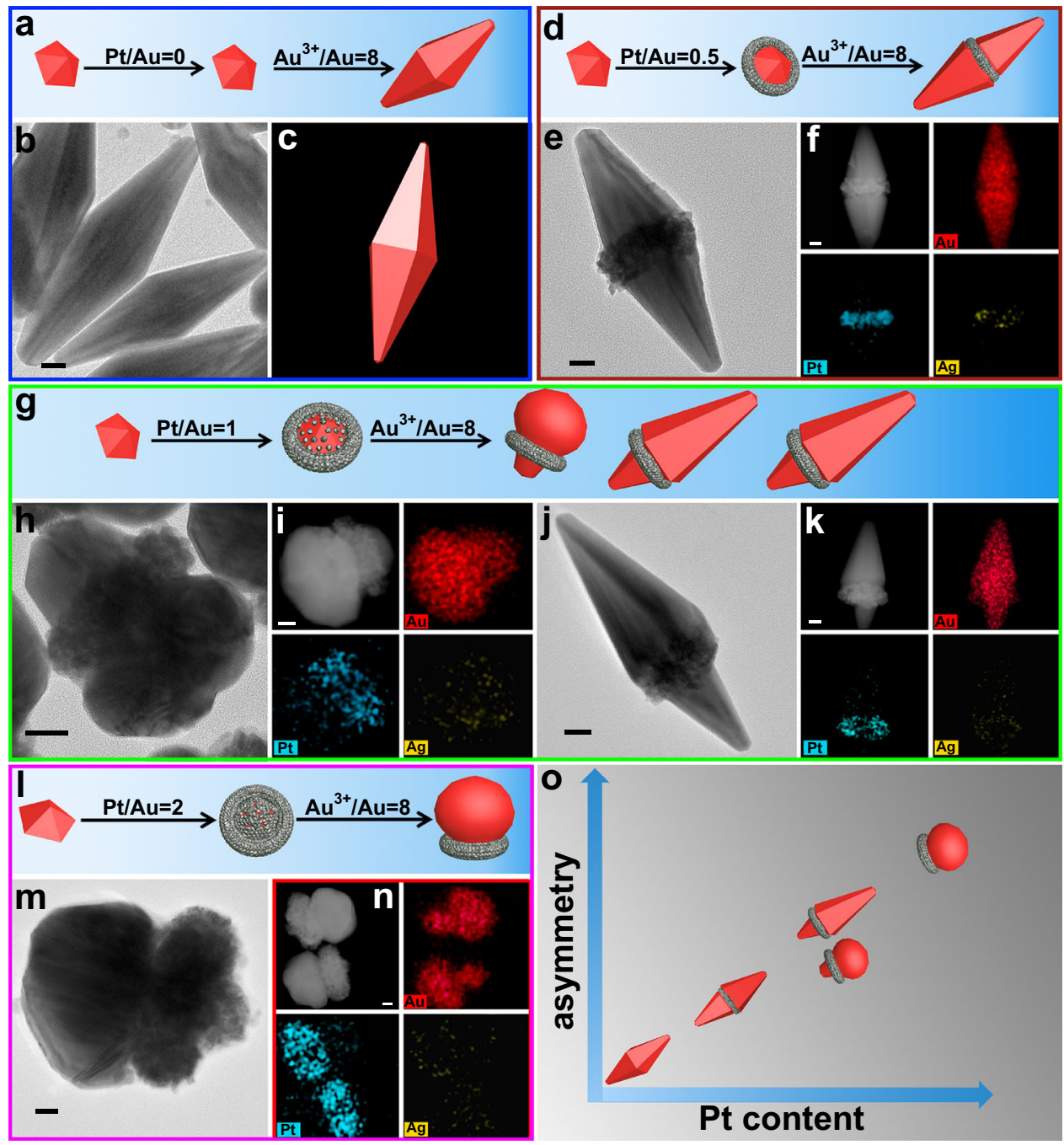

Fig. 3 Au growths on AuND-based nanocrystals. TEM images, STEM-HAADF images, STEM-EDS elemental maps, and schematic illustrations of products prepared through growing Au on AuND@PtNC nanocrystals with different molar ratios of Pt/Au: a, b, c 0; d, e, f 0.5; $, \mathbf{g}, \mathbf{i}, \mathbf{j}, \mathbf{k} 1 ; \mathbf{I}, \mathbf{m}, \mathbf{n} 2$. o Schematic evolution of spatial configuration with Pt. All scale bars are $20 \mathrm{~nm}$. In all schematics, the red and gray colors represent Au and Pt, respectively.

direction was completely blocked because Pt coating led to large lattice mismatch in these positions. Relatively, longitudinal growth along $<110>$ was promoted and produced dumbbell-like AuNR-(AuNR@Pt)-AuNR nanocrystals, meaning that Pt enhances the migration of $\mathrm{Au}$ adatoms from side surfaces to two ends during growth process. Meanwhile, two newly formed $\mathrm{Au}$ domains have no equal size, and this difference grows with increase of Pt. Further rising Pt content on AuNRs (the Pt/Au molar ratio was 0.5 ) brought about lower-symmetry unidirectional growth, yielding Janus-like (AuNR@Pt)-AuNR nanocrystals (Fig. 5j-1 and Supplementary Figs. 27-34). If the molar ratio of $\mathrm{Pt}$ and $\mathrm{Au}$ reached 1 (Fig. $5 \mathrm{~m}-\mathrm{O}$ and Supplementary Figs. 35-44), the growth pattern had no change. However, the majority of formed $\mathrm{Au}$ domains are single crystalline instead of PFT (Supplementary Fig. 39), indicating that the PFT AuNRs almost completely lost the ability to guide Au growth because of thick Pt coating. It is worth noting that the symmetry breaking has no obvious dependence on Au deposition rate. For example, when AuNRs fully coated by thick Pt layer were used as seeds, enhancing $\mathrm{Au}$ deposition rate (by increasing the molar ratio of $\mathrm{HAuCl}_{4} / \mathrm{Au}$ from 4 to 7 or 12) did not change the growth mode (Supplementary Figs. 31, 34, 38, 44); that is, growths occurred only on one side in all cases with different Au deposition rates.
Therefore, the symmetry breaking relies on directional Au adatom diffusion but not on Au deposition rate.

Above shape evolution with $\mathrm{Pt}$ is consistent with cases of $\mathrm{Au}$ growths on AuND@PtNC seeds in the following two respects: (1) $\mathrm{Pt}$ content determines the growth symmetry, and (2) formed Au domains are unable to inherit the PFT crystalline structure of AuNDs and AuNRs when Pt coating is dense. However, the minimum Pt content on AuNRs $(\mathrm{Pt} / \mathrm{Au}=0.5: 1$ molar ratio) for achieving unidirectional growth is significantly lower than that on AuNDs $(\mathrm{Pt} / \mathrm{Au}=2: 1$ molar ratio), which might be caused by the differences in the growth selectivity of Pt on their surfaces. The growth selectivity of Pt on AuND is drastically higher than that on AuNR. For this reason, full coating of AuND surface, especially these positions far from $\{100\}$ facets, requires large amount of $\mathrm{Pt}$ to realize unidirectional growth.

AuNBs are another type of common one-dimensional PFT nanocrystals which also served as seeds to test the effectiveness of surface lattice engineering. The growths of other metals on Pt-free AuNB have been reported extensively, and only symmetrical growth was observed so far ${ }^{42,62}$, but Pt-coated AuNBs seeds (Pt/ $\mathrm{Au}=0.5: 1 \mathrm{~mol} / \mathrm{mol}$ ), similar to AuND@PtNC and AuNR@Pt, could induce an asymmetrical growth in our experiment (Fig. 5p-r and Supplementary Figs. 49, 50). 


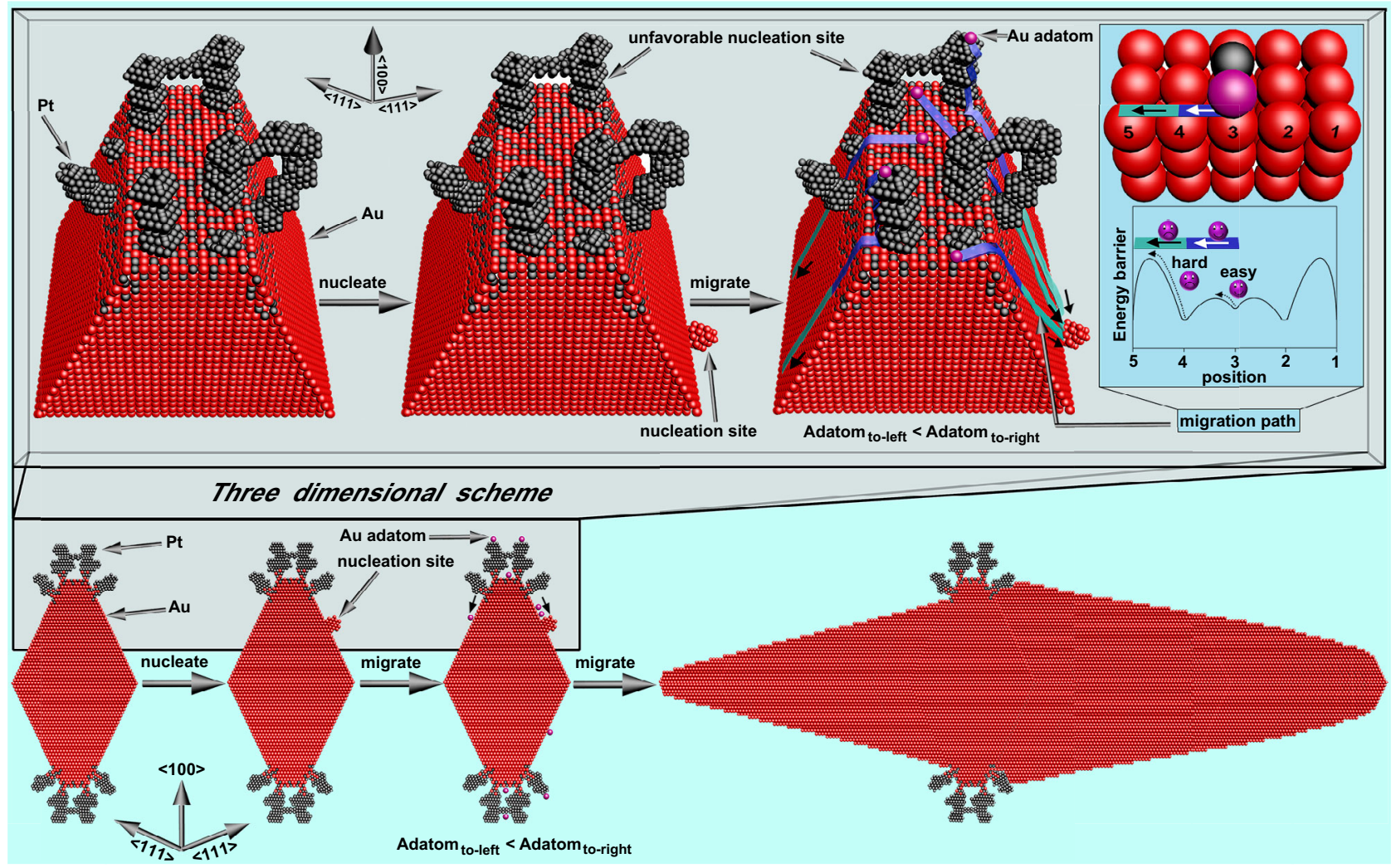

Fig. 4 Pt-dependent asymmetry. Schematic of growing asymmetrical AuNB@PtNC.

AuTNO-based nanocrystals with controlled geometrical symmetry. Above used AuND, AuNR, and AuNB are typically multitwinned nanocrystals. To further explore whether this approach has a broader applicability, single crystalline AuTNOs seeds were also tested. Results show that this strategy has no dependence on the shape and crystalline feature of seeds, and it can be used as a general approach for controlling growth pattern and tuning spatial symmetry. For example, when Pt-free AuNTOs served as seeds (Supplementary Fig. 52), spherical particles were formed (Fig. 6a-c and Supplementary Fig. 52). Well known, sphere has highest symmetry, and therefore, the growth surrounding seed is homogeneous in this case. However, when AuTNOs coated by small amount of $\mathrm{Pt}$ were used as seeds $(\mathrm{Pt} / \mathrm{Au}=0.05: 1 \mathrm{~mol} / \mathrm{mol})$, multilump nanocrystals were generated (Fig. $6 \mathrm{~d}-\mathrm{f}$ and Supplementary Figs. 53-57), implying that the growth symmetry is low relative to the growth on Pt-free AuNTOs. HRTEM images and FFT patterns show that these newly formed $\mathrm{Au}$ lumps grew along $<100>$ (Supplementary Fig. 56), which means Au adatoms selectively migrated to $\{100\}$ facet and deposited there in growth process. Moreover, the size of each lump even in same particle is significantly different, so it is known the growths are not equal on various $\{100\}$ facets. If the molar ratio of Pt and Au in AuNTO@Pt seeds was increased to 0.15 (Fig. $6 \mathrm{~g}-\mathrm{i}$ and Supplementary Figs. 58-60), the number of lumps decreased and their size difference increased, which means that the growth symmetry further reduced. When the molar ratio of $\mathrm{Pt}$ and $\mathrm{Au}$ was 0.3 or higher (Fig. 6j-1 and Supplementary Figs. 61-72), Au atoms only grew on one $\{100\}$ facets, and this extremely asymmetrical growth led to the formation of Janus-like (AuTNO@Pt)-Au nanocrystals. The above observations are well consistent with the results presented in Figs. 4, 5. Apparently, tuning growth symmetry via the surface lattice engineering approach is independent of the shape and crystalline structure of $\mathrm{Au}$ seeds.
The universality of this strategy can be further confirmed by Au growth on Pt-coated single crystalline AuNP. A few AuNPs were also formed during the preparation of AuNDs (Supplementary Fig. 77), and subsequent Pt deposition happened selectively on the $\{110\}$ facets of AuNPs, yielding AuNP@PtNC nanocrystals (Supplementary Figs. 78-80). The high growth preference of $\mathrm{Pt}$ on AuNP is due to the bounding $\{110\}$ facets which are more active than $\{111\}$ facet $^{55}$; this issue is not the main point in this article and is not discussed further. Our main concern is whether the PtNCs could play a role in determining growth symmetry. Results show that the Au growth on AuNP@PtNC followed a pattern similar to that on AuND@PtNC, and two pyramid-like $\mathrm{Au}$ domains with different sizes were formed on the top and bottom sides of AuNP (Supplementary Figs. 81-83).

Spatial composition control of complex nanocrystals and predictable synthesis. Lattice-mismatch-driven adatom migration is universal in crystal growth $39,56,63$, so this surface lattice engineering strategy can also offer practical guidance for the rational design and engineering of nanocrystals composed of other metals. Ag, Au, Pt, and $\mathrm{Pd}$ are four common noble metals, and their cell parameters are $4.086 \AA$, $4.078 \AA$, $3.924 \AA$ and $3.891 \AA$, respectively ${ }^{57}$. Ag and Pt have a larger lattice mismatch than $\mathrm{Au}$ and $\mathrm{Pt}$, so Pt-Ag interface should have a higher energy relative to Pt-Au interface, which is confirmed by DFT calculations (Supplementary Fig. 84). In other words, Pt-Au interface is more thermodynamically stable than Pt-Ag interface; as a result, $\mathrm{Ag}$ adatoms should have a stronger trend to migrate away from Pt-coated surface. The energy barrier (activation energy) for Ag adatom diffusion (migration) was also calculated under the same condition as the case of $\mathrm{Au}$ adatom (Fig. 1c, d), and result reveals that the energy barrier for Ag adatom migration is lower than that for $\mathrm{Au}$ adatom (Supplementary Fig. 85). Therefore, Ag adatom migration 
a
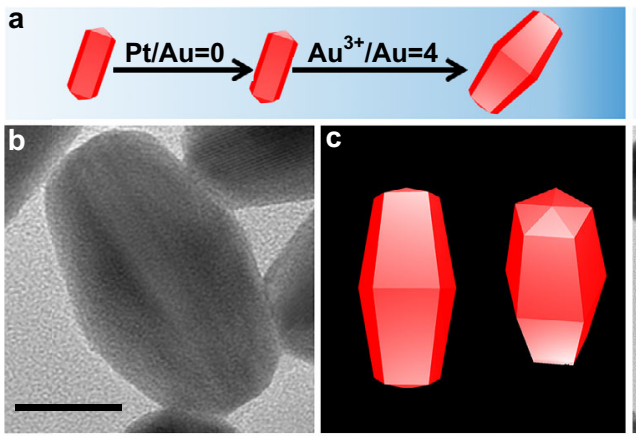

g
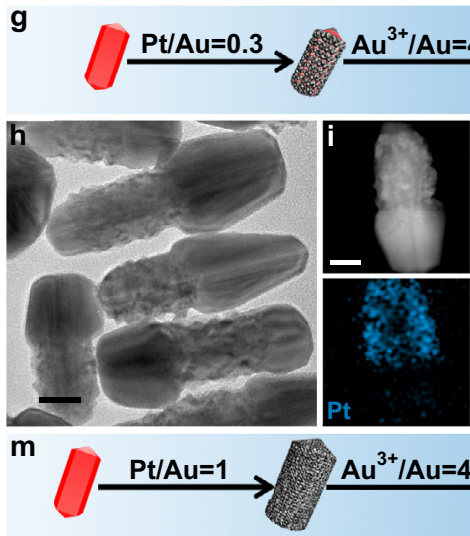

n

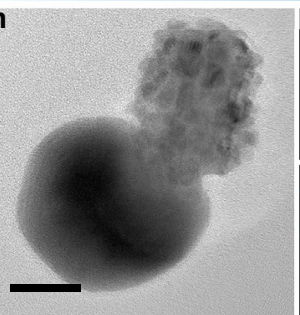

$\mathrm{Au}^{3+} / \mathrm{Au}=4$
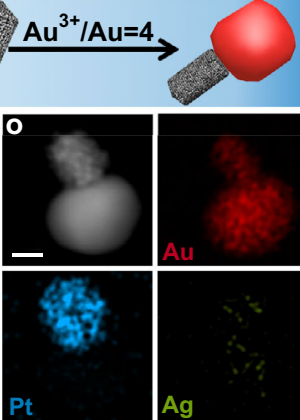
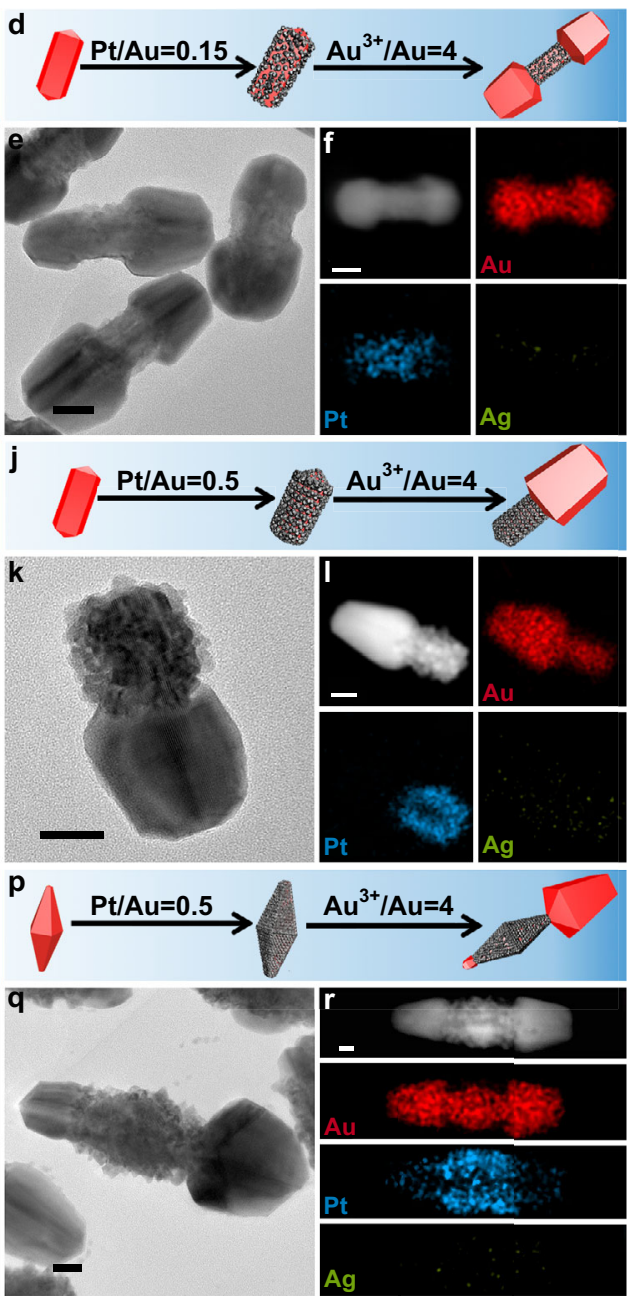

Fig. 5 Au growths on AuNR@Pt and AuNB@Pt nanocrystals. TEM images, STEM-HAADF images, STEM-EDS elemental maps, and schematic

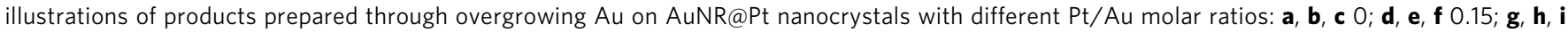

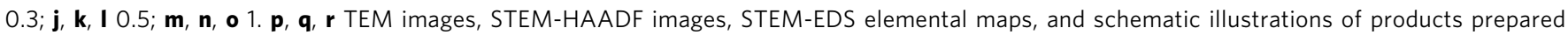
through overgrowing Au on AuNB@Pt nanocrystals. All scale bars are $20 \mathrm{~nm}$. In all schematics, the red and gray colors represent Au and Pt, respectively.

should be faster theoretically than $\mathrm{Au}$ adatom on Pt-covered $\mathrm{Au}$ surface. In fact, the Ag growths on AuNDs, AuND@PtNC nanocrystals, AuNRs, and AuNR@Pt nanocrystals (shown in Fig. 7) well agree with DFT studies. Pt-free AuNRs induced the formation of segmental AgNR-AuNR-AgNR nanocrystals in which almost all AuNRs are in the middle (Fig. 7a-c and Supplementary Figs. 86-88). As expected, AuNR@Pt seeds (Pt/Au=0.3:1 mol/mol) facilitated unidirectional growth, creating (AuNR@Pt)-AgNR nanocrystals (Fig. 7d-f and Supplementary Figs. 89-92). It has been demonstrated that Au growth on the same AuNR@Pt seeds was bidirectional along $<110>$ (Fig. 5g-i and Supplementary Fig. 26), indicating that Ag adatom indeed migrates faster than Au adatom on Pt-coated seed surface. This is further confirmed by the similar growth of $\mathrm{Ag}$ on AuND-based seeds. Pt-free AuNDs led to the formation of AgNRAuND-AgNR nanocrystals (Fig. $7 \mathrm{~g}-\mathrm{i}$ and Supplementary Figs. 93-95). However, for the case of AuND@PtNC seeds, only unidirectional growth occurred, and Janus-like (AuND@PtNC)AgNR nanocrystals were generated (Fig. 7j-1 and Supplementary Figs. 96, 97). AuND@PtNC seeds with a high Pt content resulted in irregular Ag domains instead of PFT AgNRs (Fig. 7m-o and Supplementary Figs. 98-101), because AuNDs were densely covered by $\mathrm{Pt}$ and hence incapable of effectively guiding the growth of Ag; this is similar to Au growth (Fig. 3m).
Furthermore, high-order hybrid nanocrystals with more complex structures can be obtained via rational design and predictable synthesis. For example, if the Janus-like (AuNR@Pt)AuNR nanocrystals shown in Fig. 5k acted as seeds for Ag growth, it is predictable that $\mathrm{Ag}$ adatoms should selectively migrate to Pt-free Au domain and deposit there, because Ag and $\mathrm{Pt}$ have a larger lattice mismatch than $\mathrm{Ag}$ and $\mathrm{Au}^{47,57}$. The formation of (AuNR@Pt)-AuNR-AgNR nanocrystals confirms this (Fig. 7p-r and Supplementary Figs. 102, 103). The (AuNR@Pt)-Au seeds with single crystalline $\mathrm{Au}$ domain presented in Fig. 5n guided a similar growth, yielding (AuNR@Pt)-(Au@Ag) nanocrystals (Fig. 7s-u and Supplementary Figs. 104-107). The cell parameter of Pd is close to that of Pt. If the former grows on the same (AuNR@Pt)-Au seeds, there is a high probability that $\mathrm{Pd}$ adatoms migrate to $\mathrm{Pt}$-rich positions because $\mathrm{Pt}-\mathrm{Pd}$ interface has a lower energy than $\mathrm{Pd}-\mathrm{Au}$ interface. HRTEM and elemental maps provide solid evidences that Pd atoms indeed deposited only on Pt-coated surface rather than $\mathrm{Au}$ exposed surface (Fig. 7v-x and Supplementary Figs. 108, 109).

Dimension control of nanocrystals. The above-mentioned results distinctly demonstrate that the geometrical shape and 

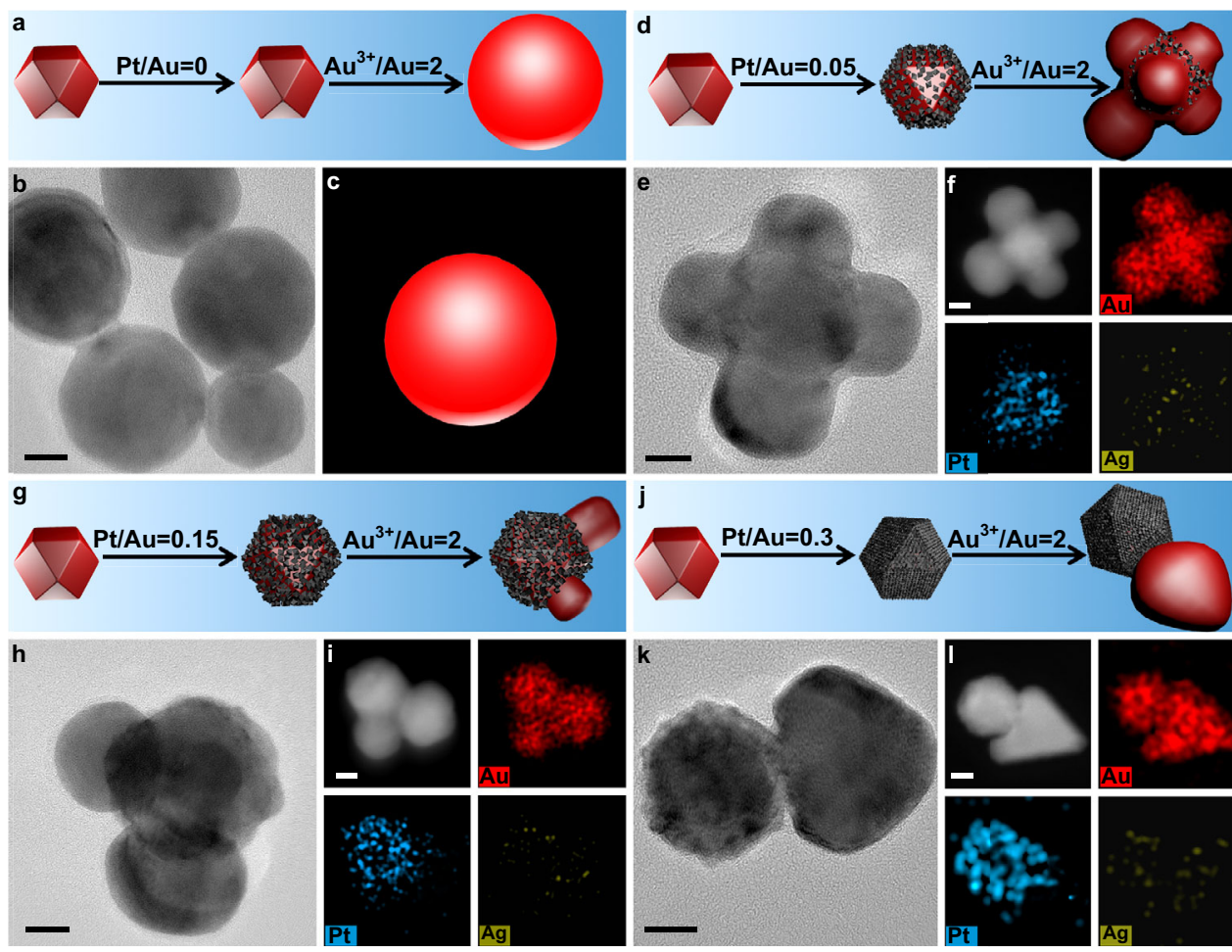

Fig. 6 Au growths on AuTNO@Pt nanocrystals. TEM images, STEM-HAADF images, STEM-EDS elemental maps, and schematic illustrations of products prepared through overgrowing Au on AuTNO@Pt nanocrystals with different molar ratios of Pt and Au: a, b, c 0; d, e, f 0.05; g, h, i 0.15; j, k, I 0.3. All scale bars are $10 \mathrm{~nm}$. In all schematics, the red and gray colors represent $\mathrm{Au}$ and Pt, respectively.

spatial composition distribution of nanocrystals can be well controlled via the surface lattice engineering strategy reported here. Moreover, this approach also allows tuning the size dimension of each section in nanocrystals. For example, when depositing Au on AuTNO@Pt seeds, adjusting the amount of $\mathrm{HAuCl}_{4}$ created various sized $\mathrm{Au}$ domains. For low-concentration precursor $\left(\mathrm{HAuCl}_{4} / \mathrm{Au}=0.2: 1\right.$ molar ratio), most of formed $\mathrm{Au}$ domains are spheres with $13 \mathrm{~nm}$ diameter (Fig. 8a-c and Supplementary Fig. $73 \mathrm{~d}-\mathrm{f}$ ). When the molar ratio of $\mathrm{HAuCl}_{4}$ and $\mathrm{Au}$ was increased to 0.4 , Au domain had no significant shape change, but their average diameter increased to $18 \mathrm{~nm}$ (Fig. $8 \mathrm{~d}-\mathrm{f}$ and Supplementary Fig. $73 \mathrm{~g}-\mathrm{i}$ ). If the molar ratio of $\mathrm{HAuCl}_{4}$ and $\mathrm{Au}$ reached 0.8 (Fig. 8g-i and Supplementary Fig. 73j-1), Au domains clearly became larger $(>22 \mathrm{~nm})$. Meanwhile, their shape is not uniform compared with the above two cases, and most are highly truncated octahedra. When the molar ratio of $\mathrm{HAuCl}_{4}$ and $\mathrm{Au}$ was 3, large sized polyhedral $\mathrm{Au}$ domains were formed (Fig. 8j-1 and Supplementary Fig. $73 \mathrm{~m}-\mathrm{O}$ ). The shape evolution of $\mathrm{Au}$ domains with $\mathrm{Au}$ precursor might be related with size effect ${ }^{64}$. For small particles, their surface atoms, as a consequence of low coordination number, are often very active, so energy minimization requires them to adopt spherical morphology with small surface area to compensate high energy. With the increase of $\mathrm{Au}$ precursor, the formation of large sized nonspherical particles is thermodynamically allowed because surface energy reduces with size. For all cases, only dimeric nanocrystals were formed even in case of high-concentration metal precursor, which implies that asymmetrical growth is caused by lattice mismatch instead of kinetics. Besides, the growths of Au domain were very fast and almost completed within $5 \mathrm{~min}$ in all syntheses, which does not match the typical characteristics of slow growth rate required to prepare dimeric nanocrystals using kinetic-controlled process ${ }^{65}$.

As well as the formed Au domains, AuTNO seeds can be tuned in size. That is, this strategy also has no dependence on the size of
$\mathrm{Au}$ seeds. For example, the AuTNO seeds for synthesizing nanocrystals shown in Fig. 8 have $24 \mathrm{~nm}$ edge length. When Ptcoated large AuTNOs with $54 \mathrm{~nm}$ edge length were used as seeds, a similar growth mode was observed and also created Janus-like (AuTNO@Pt)-Au nanocrystals (Supplementary Figs. 74-76). Such control is also suited to the case of PFT Au seeds (Supplementary Figs. 22, 23, 30, 31, 34, 37, 38, 44, 45e, 46), in which the length of AuNRs seeds was tunable and the size of formed $\mathrm{Au}$ domain could be easily controlled through introducing different amount of $\mathrm{HAuCl}_{4}$.

Multifunctional nanocrystals integrating surface enhanced Raman scattering (SERS) and catalytic activity. In addition to the fine control over spatial configuration, multi-functionalization of nanocrystals also can be achieved using this surface lattice engineering strategy. For example, AuNBs have excellent SERS performance because their sharp tips (hot spots) leads to a larger enhancement of local electric field relative to other $\mathrm{Au}$ nanocrystals ${ }^{42,66-68}$, but they are often inactive in chemical reactions ${ }^{14}$. On the contrary, Pt nanocrystals have high catalytic activity but very poor SERS performance ${ }^{54,69,70}$. Combination of both advantages into a single hybrid nanosystem is evidently attractive, which however cannot be realized with conventional approaches. In conventional synthesis, AuNBs are pre-synthesized, followed by Pt deposition; despite improved catalytic performance, SERS activity of AuNBs greatly reduces because of $\mathrm{Pt}$ coating on their sharp tips ${ }^{71}$. Interestingly, such combination is easily implemented in the synthesis of AuNB@PtNC nanocrystals. Surface lattice engineering requires that $\mathrm{Pt}$ deposition is ahead of the growth of AuNBs. This reverse arrangement completely eliminates the effect of Pt on SERS activity, as demonstrated by the following SERS measurements. 4-nitrothiophenol (4-NTP) probe molecules on AuNB@PtNCs exhibit a strong 

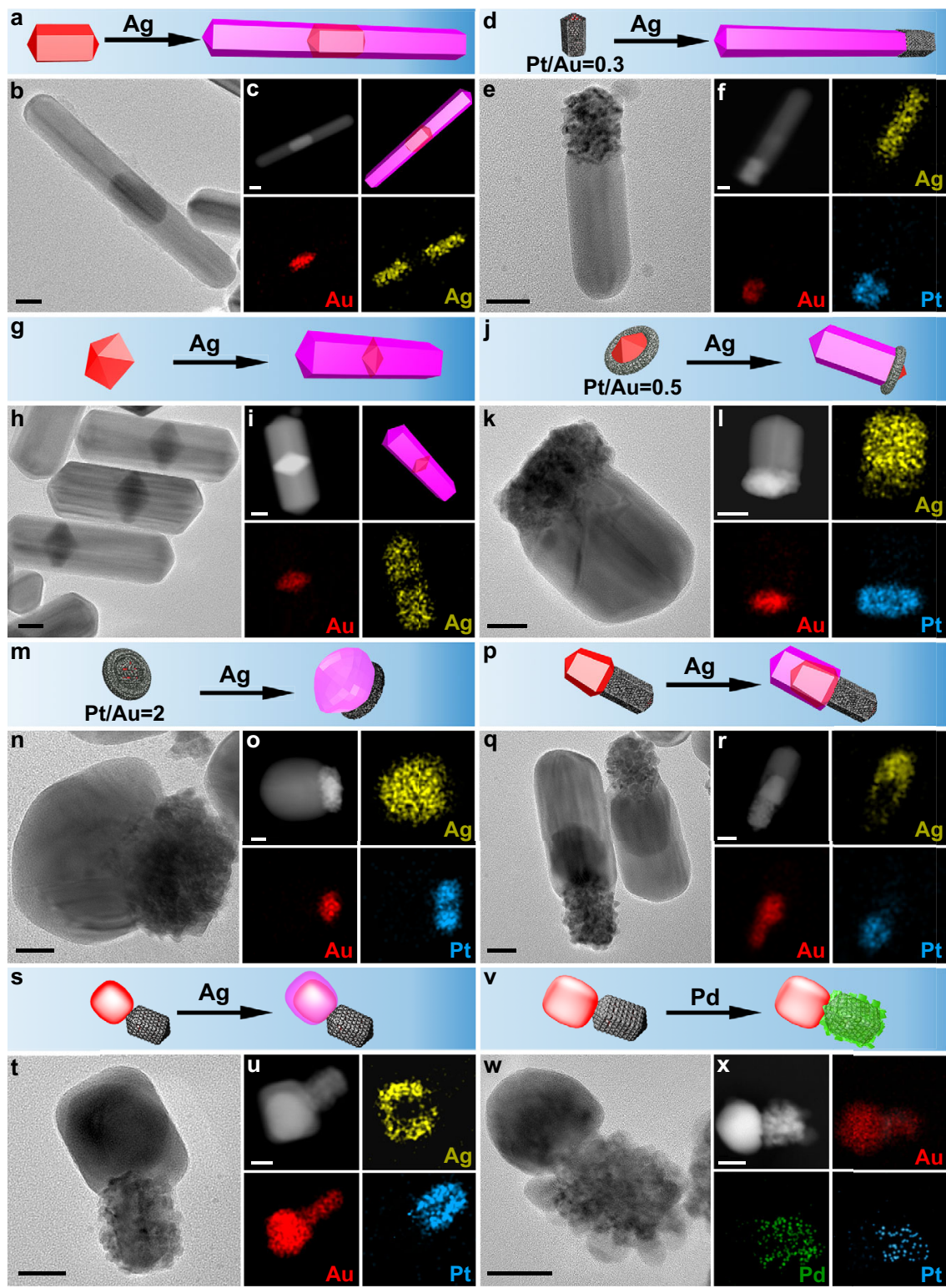

Fig. 7 Nanocrystals syntheses with tuned spatial composition. TEM images, STEM-HAADF images, STEM-EDS elemental maps, and schematic illustrations of products prepared through growing Ag on various seeds: a, b, c AuNR; d, e, f AuNR@Pt (the molar ratio of Pt and Au was 0.3); $\mathbf{g}$, h, i AuND; j, $\mathbf{k}$, I AuND@PtNC (the molar ratio of Pt and Au was 0.5); $\mathbf{m}, \mathbf{n}, \mathbf{o}$ AuND@PtNC (the molar ratio of Pt and Au was 2); $, \mathbf{q}, \mathbf{q}$ nanocrystals shown in 5k; $\mathbf{5}, \mathbf{t}$, $\mathbf{u}$ nanocrystals shown in $\mathbf{5 n}$. v, w, x TEM images, STEM-HAADF images, STEM-EDS elemental maps, and schematic illustrations of products prepared through growing Pd on nanocrystals shown in $\mathbf{5 n}$. All scale bars are $20 \mathrm{~nm}$. In all schematics, the red, gray, mauve, and green colors represent $\mathrm{Au}$, Pt, $\mathrm{Ag}$, and $\mathrm{Pd}$, respectively.

Raman signal comparable to that on AuNBs (Fig. 9a). However, the Raman signal recorded on (AuNB-core)@(Pt-shell) nanocrystals prepared with conventional method is five times weaker. Electric field enhancement simulations further confirm above result (Fig. 9b-d). In situ monitoring the catalytic reduction of 4-NTP to 4-aminothiolphenol (4-ATP) with SERS demonstrates that AuNB@PtNC nanocrystals also have enhanced catalytic activity thanks to the presence of PtNCs. For AuNB@PtNC catalyst, the spectra band corresponding to 4-NTP gradually decreased with time (Fig. 9e-g). Three new Raman bands of intermediate $\left(4,4^{\prime}\right.$-dimercapto-azobenzene) at 1143,1388 and $1430 \mathrm{~cm}^{-1}$ were observed after $2 \mathrm{~min} ; ;^{14,20,54}$ these bands gradually increased, then progressively decreased, and entirely disappeared after $26 \mathrm{~min}$. In addition, two new bands (1490 and $1600 \mathrm{~cm}^{-1}$ ) attributable to 4-ATP appeared. Their intensity continued to increase and then became stable after $26 \mathrm{~min}$.
Apparently, the reaction between 4-NTP and sodium borohydride (reducing agent) was very fast, and was completed within 26 minutes. However, for the AuNB catalyst, almost no change was observed due to its low catalytic activity even after $44 \mathrm{~min}$ (Fig. 9h-j). The improved catalytic performance of the AuNB@PtNC nanocrystals mainly stems from the introduction of active Pt component, and furthermore, the synergistic effect of two metals also might make contributions. Pt-on-Au structure can result in electron donation from $\mathrm{Pt}$ to $\mathrm{Au}$, which increases the $5 \mathrm{~d}$ vacancies in $\mathrm{Pt}$ and hence facilitates the absorption of reactant with electron donor group ${ }^{72}$. Thus, the reaction is promoted. In addition, electron transfer increases the electron density on $\mathrm{Au}$, and 4-NTP obtains electrons more easily; therefore, the catalytic activity of $\mathrm{Au}$ is also improved ${ }^{52}$. Deeply understanding this needs a great deal of research and will be targeted to other topics. This example was mainly used to demonstrate that advantages of 

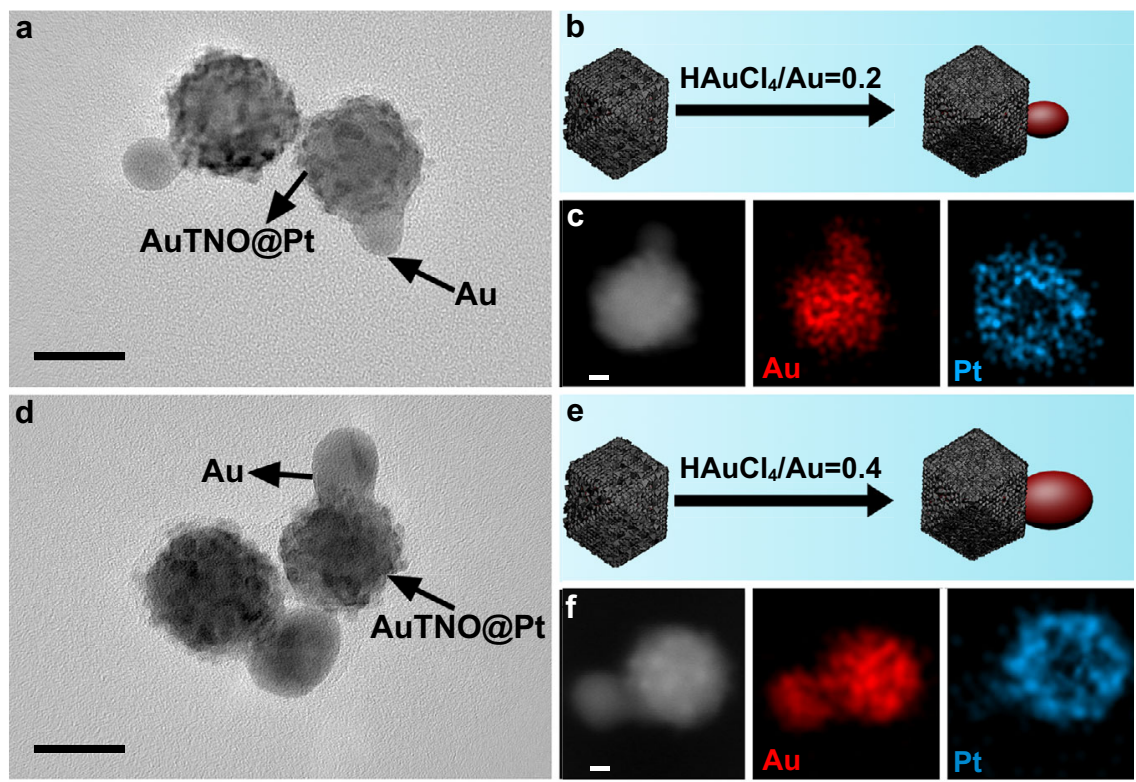

e
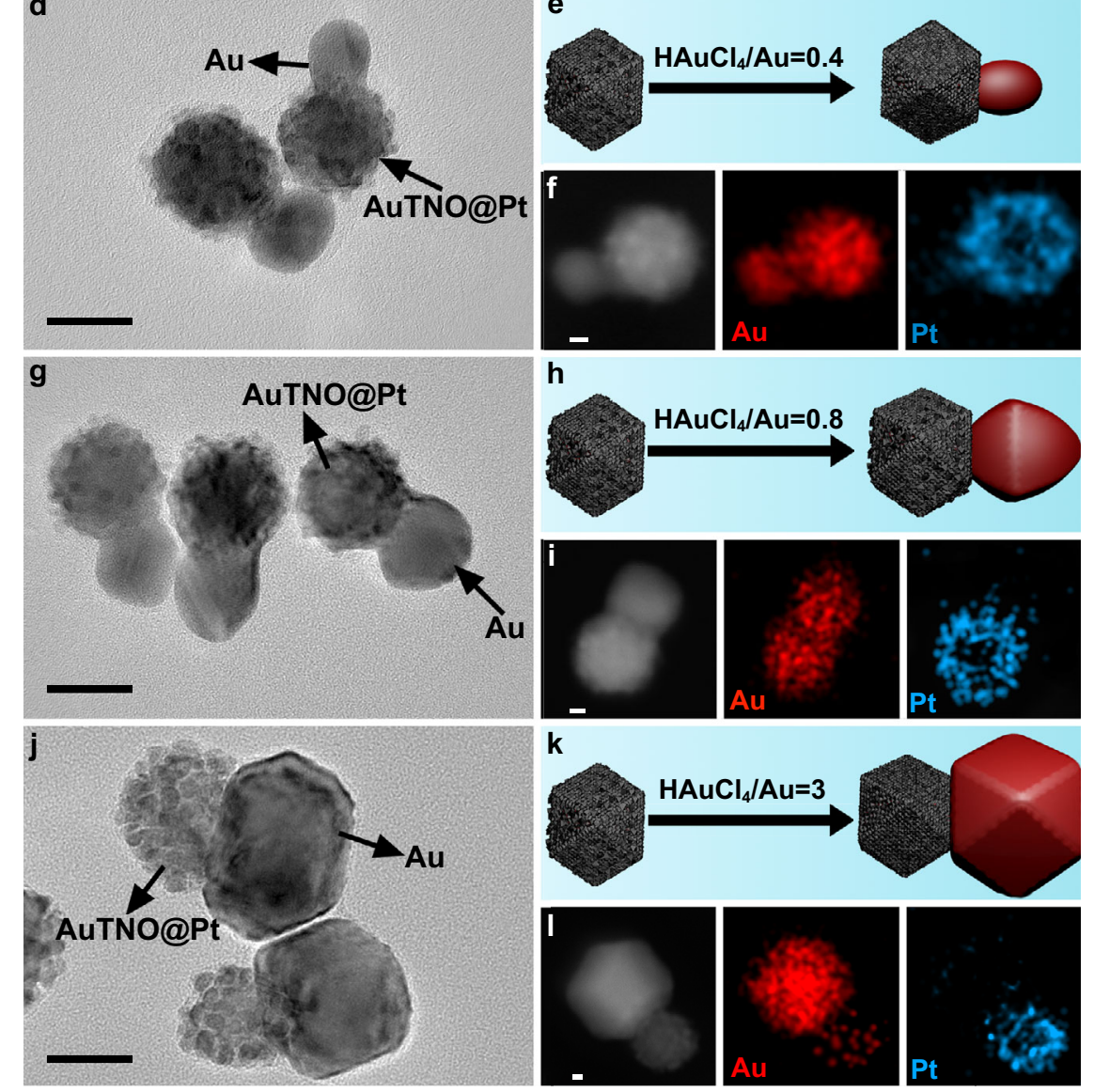

Fig. 8 Dimension control of nanocrystals. TEM images, schematic illustrations, and elemental maps of products prepared through tuning the molar ratio of $\mathrm{HAuCl}_{4}$ and Au when using AuTNO@Pt nanocrystals as seeds: a, b, c 0.2; d, e, f $0.4 ; \mathbf{g}, \mathbf{h}, \mathbf{i} 0.8 ; \mathbf{j}, \mathbf{k}, \mathbf{I} 3$. Scale bars in a, $\mathbf{d}, \mathbf{g}$, jare $20 \mathrm{~nm}$. Scale bars in $\mathbf{c}, \mathbf{f}, \mathbf{i}$, I are $5 \mathrm{~nm}$. In all schematics, the red and gray colors represent $\mathrm{Au}$ and $\mathrm{Pt}$, respectively.

various materials can be integrated together via surface lattice engineering.

\section{Discussion}

In summary, we developed a surface lattice engineering strategy for precisely tuning the growth pattern of seeded growth and constructing hybrid nanocrystals with fine-tuned spatial configuration in shape, composition, and dimension. A variety of hybrid nanocrystals unaccessible to conventional synthetic technologies can be prepared in a controlled manner. Furthermore, the results presented in this work also may provide a potential approach for rationally designing and predictably synthesizing hybrid nanocrystals with high complexity and optimized structure. Two critical factors of this approach, namely lattice mismatch and adatom diffusion, are universal phenomena in hybrid crystalline composites, including metals, oxide metals, semiconductors, and even organic crystals ${ }^{25,38,73-75}$. Presumably, this strategy can be generalized and extended to various other crystalline material systems to gain broader applications.

\section{Methods}

Preparation of AuNDs. A total of $12.5 \mu \mathrm{L}$ aqueous chloroauric acid $\left(\mathrm{HAuCl}_{4}\right.$, $0.48 \mathrm{M}$ ) was transferred into a $50-\mathrm{mL}$ beaker which was then heated in $125^{\circ} \mathrm{C}$ oven. After water was removed completely, $10 \mathrm{~mL}$ diethylene glycol (DEG) containing $0.25 \mathrm{~mL}$ poly (diallyldimethylammonium chloride) (PDDA, $\mathrm{Mw}=400000-500000$ $20 \mathrm{wt} \%$ in $\mathrm{H}_{2} \mathrm{O}$ ) was added, and then the solution was stirred vigorously until a yellow homogeneous solution was formed. $3 \mathrm{~mL} \mathrm{DEG}$ containing $6 \mathrm{mg}$ silver nitrate $\left(\mathrm{AgNO}_{3}\right)$ was introduced into above yellow solution. The mixture was stirred for another 3 minutes and then transferred into $25-\mathrm{mL}$ round-bottom flask which was then heated in $200{ }^{\circ} \mathrm{C}$ oil bath. After $30 \mathrm{~min}$, the red solution was cooled down to room temperature, and then $12 \mathrm{~mL}$ water was added to form seed solution for preparing AuND@PtNCs.

Preparations of AuND@PtNCs. In total $10 \mathrm{~mL}$ above seed solution was heated to $80^{\circ} \mathrm{C}$, and then aqueous chloroplatinic acid $\left(\mathrm{H}_{2} \mathrm{PtCl}_{6}, 19.3 \mathrm{mM}\right)$ and ascorbic acid $(\mathrm{AA}, 56.8 \mathrm{mM})$ solution were introduced $\left(\mathrm{AA} / \mathrm{H}_{2} \mathrm{PtCl}_{6}=2: 1 \mathrm{~mol} / \mathrm{mol} ; \mathrm{Pt} / \mathrm{Au}=1: 2\right.$ $\mathrm{mol} / \mathrm{mol}$ ). After $10 \mathrm{~h}$ reaction, a gray solution containing AuND@PtNCs was cooled to room temperature and could be used as seed solution for preparing AuNB@PtNC 

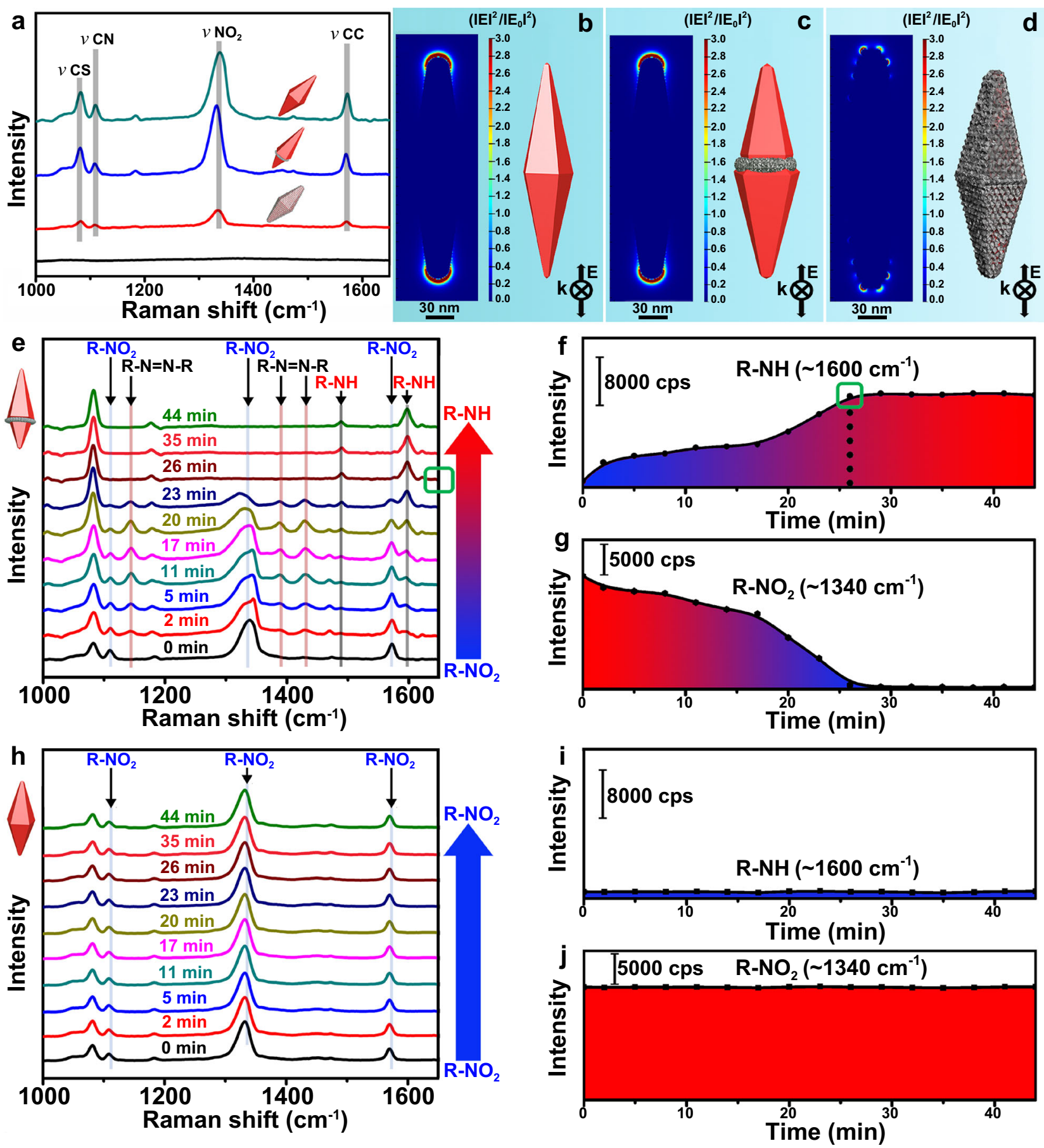

Fig. 9 Optical properties and in situ monitoring organic reaction with SERS. a Raman spectra recorded on AuNB@PtNC (longitudinal length: $236 \mathrm{~nm}$. Transverse length: $72 \mathrm{~nm}$ ), AuNB (longitudinal length: $230 \mathrm{~nm}$. Transverse length: $70 \mathrm{~nm}$ ) and (AuNB-core)@(Pt-shell) nanocrystals. b, c, d Electrical field enhancement on the surface of various nanocrystals. Raman spectra, peak intensity of R-NO $\mathrm{NO}_{2}$ at $1340 \mathrm{~cm}^{-1}$ and peak intensity at $1600 \mathrm{~cm}-1$ with time when using e, f, g AuNB@PtNC nanocrystals and $\mathbf{h}, \mathbf{i}, \mathbf{j}$ AuNBs as catalysts. In all schematics, the red and gray colors represent Au and Pt, respectively. Source data are provided as a Source Data file.

nanocrystals. To control the thickness of Pt coating, various concentrations of $\mathrm{H}_{2} \mathrm{PtCl}_{6}$ and $\mathrm{AA}$ were introduced, while the molar ratio of $\mathrm{AA} / \mathrm{H}_{2} \mathrm{PtCl}_{6}$ was kept unchanged.

Preparations of AuNB@PtNC nanocrystals. A total of $10 \mu \mathrm{L}$ ammonia water (20\% volume ratio; freshly prepared ammonia water is highly recommended) and an aqueous $\mathrm{HAuCl}_{4}$ solution $(0.048 \mathrm{M})$ were added into $1 \mathrm{~mL}$ AuND@PtNC seed solution $\left(\mathrm{Au}^{3+} / \mathrm{Au}=10 \mathrm{~mol} / \mathrm{mol}\right)$. After vigorous shaking for $30 \mathrm{~s}$, the mixture was sealed in $50 \mathrm{~mL}$ glass pressure tube $(19 \mathrm{~cm}$ in length, $2.6 \mathrm{~cm}$ in outer diameter, and
$4 \mathrm{~mm}$ wall thickness), and then it was quickly moved into a $120^{\circ} \mathrm{C}$ oil bath. After 20 min, AuNB@PtNC nanocrystals could be formed.

More details of other syntheses and characterizations can be seen in Supplementary methods.

\section{Data availability}

All data generated in this study are provided within the manuscript and the Supplementary Information. Source data are provided with this paper Source data are provided with this paper. 
Received: 20 December 2020; Accepted: 2 September 2021; Published online: 27 September 2021

\section{References}

1. Luo, M. et al. PdMo bimetallene for oxygen reduction catalysis. Nature 574, 81-85 (2019).

2. Tian, X. et al. Engineering bunched Pt-Ni alloy nanocages for efficient oxygen reduction in practical fuel cells. Science 366, 850-856 (2019).

3. Xiong, L. et al. Octahedral gold-silver nanoframes with rich crystalline defects for efficient methanol oxidation manifesting a CO-promoting effect. Nat. Commun. 10, 3782 (2019).

4. Yang, P., Zheng, J., Xu, Y., Zhang, Q. \& Jiang, L. Colloidal synthesis and applications of plasmonic metal nanoparticles. Adv. Mater. 28, 10508-10517 (2016).

5. Zhu, J. et al. Pt-Ir-Pd trimetallic nanocages as a dual catalyst for efficient oxygen reduction and evolution reactions in acidic media. Adv. Energy Mater. 10, 1904114 (2020)

6. Huang, X. et al. High-performance transition metal-doped $\mathrm{Pt}_{3} \mathrm{Ni}$ octahedra for oxygen reduction reaction. Science 348, 1230-1234 (2015).

7. Hong, J. W., Wi, D. H., Lee, S.-U. \& Han, S. W. Metal-semiconductor heteronanocrystals with desired configurations for plasmonic photocatalysis. J. Am. Chem. Soc. 138, 15766-15773 (2016).

8. Long, R., Li, Y., Song, L. \& Xiong, Y. Coupling solar energy into reactions: materials design for surface plasmon-mediated catalysis. Small 11, 3873-3889 (2015).

9. Fennell, J. et al. A selective blocking method to control the overgrowth of Pt on Au nanorods. J. Am. Chem. Soc. 135, 6554-6561 (2013).

10. Wang, D. et al. Structurally ordered intermetallic platinum-cobalt core-shell nanoparticles with enhanced activity and stability as oxygen reduction electrocatalysts. Nat. Mater. 12, 81-87 (2013).

11. Espinosa, A. et al. Cancer cell internalization of gold nanostars impacts their photothermal efficiency in vitro and in vivo: toward a plasmonic thermal fingerprint in tumoral environment. Adv. Healthc. Mater. 5, 1040-1048 (2015).

12. Li, J. F. et al. Shell-isolated nanoparticle-enhanced Raman spectroscopy. Nature 464, 392-395 (2010)

13. DeSantis, C. J., Weiner, R. G., Radmilovic, A., Bower, M. M. \& Skrabalak, S. E. Seeding bimetallic nanostructures as a new class of plasmonic colloids. J. Phys. Chem. Lett. 4, 3072-3082 (2017).

14. Zhu, X. et al. Gold nanobipyramid-embedded ultrathin metal nanoframes for in situ monitoring catalytic reactions. Chem. Sci. 11, 3198-3207 (2020).

15. Meng, M. et al. Integration of kinetic control and lattice mismatch to synthesize Pd@AuCu core-shell planar tetrapods with size-dependent optical properties. Nano Lett. 16, 3036-3041 (2016).

16. $\mathrm{Li}, \mathrm{M}$. et al. Ultrafine jagged platinum nanowires enable ultrahigh mass activity for the oxygen reduction reaction. Science 354, 1414-1419 (2016).

17. Jia, J. et al. Fine-tuning the homometallic interface of Au-on-Au nanorods and their photothermal therapy in the NIR-II window. Angew. Chem. Int. Ed. 59, 14443-14448 (2020).

18. de Aberasturi, D. J., Serrano-Montes, A. B. \& Liz-Marzán, L. M. Modern applications of plasmonic nanoparticles: from energy to health. Adv. Opt. Mater. 3, 602-617 (2015).

19. He, T. et al. Inflating hollow nanocrystals through a repeated Kirkendall cavitation process. Nat. Commun. 8, 1261 (2017).

20. Li, J., Liu, J., Yang, Y. \& Qin, D. Bifunctional Ag@Pd-Ag nanocubes for highly sensitive monitoring of catalytic reactions by surface-enhanced Raman spectroscopy. J. Am. Chem. Soc. 137, 7039-7042 (2015).

21. Zhang, L. et al. Platinum-based nanocages with subnanometer-thick walls and well-defined, controllable facets. Science 349, 412-416 (2015).

22. Oh, M. H. et al. Design and synthesis of multigrain nanocrystals via geometric misfit strain. Nature 577, 359-363 (2020).

23. Yao, Y. et al. Modulating fcc and hcp ruthenium on the surface of palladiumcopper alloy through tunable lattice mismatch. Angew. Chem. Int. Ed. 128, 5591-5595 (2016).

24. Shahjamali, M. M. et al. $\mathrm{Ag}-\mathrm{Ag}_{2} \mathrm{~S}$ hybrid nanoprisms: structural versus plasmonic evolution. ACS Nano 10,5362-5373 (2016).

25. Fan, Z. \& Zhang, H. Template synthesis of noble metal nanocrystals with unusual crystal structures and their catalytic applications. Acc. Chem. Res. 49, 2841-2850 (2016).

26. Carbone, L. \& Cozzoli, P. D. Colloidal heterostructured nanocrystals Synthesis and growth mechanisms. Nano Today 5, 449-493 (2010).

27. Huang, J. et al. Structural sensitivities in bimetallic catalysts for electrochemical $\mathrm{CO}_{2}$ reduction revealed by $\mathrm{Ag}-\mathrm{Cu}$ nanodimers. J. Am. Chem. Soc. 141, 2490-2499 (2019).
28. Lyu, Z. et al. Kinetically controlled synthesis of Pd-Cu Janus nanocrystals with enriched surface structures and enhanced catalytic activities toward $\mathrm{CO}_{2}$ reduction. J. Am. Chem. Soc. 143, 149-162 (2021).

29. Lin, M., Kim, G.-H., Kim, J.-H., Oh, J.-W. \& Nam, J.-M. Transformative heterointerface evolution and plasmonic tuning of anisotropic trimetallic nanoparticles. J. Am. Chem. Soc. 139, 10180-10183 (2017).

30. Shao, W. et al. Symmetry breaking in monometallic nanocrystals toward broadband and direct electron transfer enhanced plasmonic photocatalysis. Adv. Funct. Mater. 31, 2006738 (2020).

31. Luo, M. et al. Facile synthesis of Ag nanorods with no plasmon resonance peak in the visible region by using Pd decahedra of $16 \mathrm{~nm}$ in size as seeds. ACS Nano 9, 10523-10532 (2015).

32. Luo, M. et al. Penta-twinned copper nanorods: facile synthesis via seedmediated growth and their tunable plasmonic properties. Adv. Funct. Mater. 26, 1209-1216 (2016).

33. Krug, M. et al. Core-shell nanocuboid dimers with nanometric gaps. J. Phys. Chem. C 124, 18690-18697 (2020).

34. Zhang, J. T., Tang, Y., Weng, L. \& Ouyang, M. Versatile strategy for precisely tailored core@shell nanostructures with single shell layer accuracy: the case of metallic shell. Nano Lett. 9, 4061-4065 (2009).

35. Gilroy, K. D., Peng, H.-C., Yang, X., Ruditskiy, A. \& Xia, Y. Symmetry breaking during nanocrystal growth. Chem. Commun. 53, 4530-4541 (2017).

36. Xia, Y., Gilroy, K. D., Peng, H.-C. \& Xia, X. Seed-mediated growth of colloidal metal nanocrystals. Angew. Chem. Int. Ed. 56, 60-95 (2017).

37. Peng, Z. \& Yang, H. Synthesis and oxygen reduction electrocatalytic property of Pt-on-Pd bimetallic heteronanostructures. J. Am. Chem. Soc. 131, 7542-7543 (2009)

38. Wang, X. et al. Palladium-platinum core-shell icosahedra with substantially enhanced activity and durability towards oxygen reduction. Nat. Commun. 6 , 7594 (2015)

39. Liu, J. \& Zhang, J. Nanointerface chemistry: Lattice-mismatch-directed synthesis and application of hybrid nanocrystals. Chem. Rev. 120, 2123-2170 (2020).

40. Ye, X. et al. Seeded growth of monodisperse gold nanorods using bromide-free surfactant mixtures. Nano Lett. 13, 2163-2171 (2013).

41. Personick, M. L. \& Mirkin, C. A. Making sense of the mayhem behind shape control in the synthesis of gold nanoparticles. J. Am. Chem. Soc. 135, 18238-18247 (2013).

42. Chow, T. H. et al. Gold nanobipyramids: an emerging and versatile type of plasmonic nanoparticles. Acc. Chem. Res. 52, 2136-2146 (2019).

43. Sánchez-Iglesias, A. et al. High-yield seeded growth of monodisperse pentatwinned gold nanoparticles through thermally induced seed twinning. $J$. Am. Chem. Soc. 139, 107-110 (2017).

44. Lee, H.-E. et al. Cysteine-encoded chirality evolution in plasmonic rhombic dodecahedral gold nanoparticles. Nat. Commun. 11, 263 (2020).

45. Wang, Q. et al. Controlled growth and shape-directed self-assembly of gold nanoarrows. Sci. Adv. 3, e1701183 (2017)

46. Yu, Y., Zhang, Q., Xie, J. \& Lee, J. Y. Engineering the architectural diversity of heterogeneous metallic nanocrystals. Nat. Commun. 4, 1454 (2013).

47. Habas, S. E., Lee, H., Radmilovic, V., Somorjai, G. A. \& Yang, P. Shaping binary metal nanocrystals through epitaxial seeded growth. Nat. Mater. 6 692-697 (2007)

48. García-Negrete, C. A. et al. Shape-defined nanodimers by tailored heterometallic epitaxy. Nanoscale 6, 11090-11097 (2014).

49. Wang, C. et al. Rational synthesis of heterostructured nanoparticles with morphology control. J. Am. Chem. Soc. 132, 6524-6529 (2010).

50. Jang, H. J. et al. Fabrication of $2 \mathrm{D}$ Au nanorings with Pt framework. J. Am. Chem. Soc. 136, 17674-17680 (2014).

51. Bian, T. et al. Epitaxial growth of twinned Au-Pt core-Shell star-shaped decahedra as highly durable electrocatalysts. Nano Lett. 15, 7808-7815 (2015)

52. Zheng, Z., Tachikawa, T. \& Majima, T. Single-particle study of Pt-modified $\mathrm{Au}$ nanorods for plasmon-enhanced hydrogen generation in visible to nearinfrared region. J. Am. Chem. Soc. 136, 6870-6873 (2014).

53. Zhang, G. R. et al. Catalytic Pt-on-Au nanostructures: why Pt becomes more active on smaller Au particles. ACS Nano 6, 2226-2236 (2012).

54. Xie, W., Herrmann, C., Kömpe, K., Haase, M. \& Schlücker, S. Synthesis of bifunctional $\mathrm{Au} / \mathrm{Pt} / \mathrm{Au}$ core/shell nanoraspberries for in situ SERS monitoring of platinum-catalyzed reactions. J. Am. Chem. Soc. 133, 19302-19305 (2011).

55. Zhang, L. et al. Synthesis of convex hexoctahedral palladium@gold core-shell nanocrystals with $\{431\}$ high-index facets with remarkable electrochemiluminescence activities. ACS Nano 8, 5953-5958 (2014).

56. Schroeder, M. \& Wolf, D. E. Diffusion on strained surfaces. Surf. Sci. 375, 129-140 (1997)

57. Fan, F. R. et al. Epitaxial growth of heterogeneous metal nanocrystals: from gold nano-octahedra to palladium and silver nanocubes. J. Am. Chem. Soc. 130, 6949-6951 (2008).

58. Zhou, G. et al. Growth of nanobipyramid by using large sized Au decahedra as seeds. ACS Appl. Mater. Interfaces 5, 13340-13352 (2013). 
59. Han, S. et al. The alloying effect and $\mathrm{AgCl}$-directing growth for synthesizing a trimetallic nanoring with improved SERS. Nanoscale 7, 20414-20425 (2015).

60. Zhao, J., Chen, B. \& Wang, F. Shedding light on the role of misfit strain in controlling core-shell nanocrystals. Adv. Mater. 32, 2004142 (2020).

61. Peng, H.-S., Park, J., Zhang, L. \& Xia, Y. Toward a quantitative understanding of symmetry reduction involved in the seed-mediated growth of Pd nanocrystals. J. Am. Chem. Soc. 137, 6643-6652 (2015).

62. Lee, J. H., Gibson, K. J., Chen, G. \& Weizmann, Y. Bipyramid-templated synthesis of monodisperse anisotropic gold nanocrystals. Nat. Commun. $\mathbf{6}$, 7571 (2015).

63. Williams, B. P. et al. Strain-enhanced metallic intermixing in shape-controlled multilayered core-shell nanostructures: toward shaped intermetallics. Angew. Chem. Int. Ed. 59, 10574-10580 (2020).

64. Lu, H. M., Li, P. Y., Cao, Z. H. \& Meng, X. K. Size-, shape-, and dimensionality-dependent melting temperatures of nanocrystals. J. Phys. Chem. C 113, 7598-7602 (2009).

65. Kim, D. Y. et al. Synthesis of gold nano-hexapods with controllable arm lengths and their tunable optical properties. Angew. Chem. Int. Ed. 50, 6328-6331 (2011).

66. Shi, Q. et al. Two-dimensional bipyramid plasmonic nanoparticle liquid crystalline superstructure with four distinct orientational packing orders. ACS Nano 10, 967-976 (2016).

67. Niu, W., Duan, Y., Qing, Z., Huang, H. \& Lu, X. Shaping gold nanocrystals in dimethyl sulfoxide: toward trapezohedral and bipyramidal nanocrystals enclosed by $\{311\}$ facets. J. Am. Chem. Soc. 139, 5817-5826 (2017).

68. Zhang, Q., Large, N. \& Wang, H. Gold nanoparticles with tipped surface structures as substrates for single-particle surface-enhanced Raman spectroscopy: Concave nanocubes, nanotrisoctahedra, and nanostars. J. Am. Chem. Soc. 142, 15412-15419 (2020).

69. Chen, C. et al. Highly crystalline multimetallic nanoframes with threedimensional electrocatalytic surfaces. Science 343, 1339-1343 (2014).

70. Li, H.-H. et al. Scalable bromide-triggered synthesis of Pd@Pt core-shell ultrathin nanowires with enhanced electrocatalytic performance toward oxygen reduction reaction. J. Am. Chem. Soc. 137, 7862-7868 (2015).

71. Yoo, S. et al. Three-dimensional gold nanosphere hexamers linked with metal bridges: Near-field focusing for single particle surface enhanced Raman scattering. J. Am. Chem. Soc. 142, 15412-15419 (2020).

72. Ye, F. et al. Heterogeneous Au-Pt nanostructures with enhanced catalytic activity toward oxygen reduction. Dalton Trans. 41, 2898-2903 (2012).

73. Huang, Z. Q., Gong, J. L. \& Nie, Z. H. Symmetry-breaking synthesis of multicomponent nanoparticles. Acc. Chem. Res. 52, 1125-1133 (2019).

74. Buck, M. R., Bondi, J. F. \& Schaak, R. E. A total-synthesis framework for the construction of high-order colloidal hybrid nanoparticles. Nat. Chem. 2, 37-44 (2012).

75. Liu, C. et al. Ternary MOF-on-MOF heterostructures with controllable architectural and compositional complexity via multiple selective assembly. Nat. Commun. 11, 4971 (2020).

\section{Acknowledgements}

This work was supported by the NSFC $(5217010637,21471117)$. A portion of this work was conducted at Argonne National Laboratory. Argonne National Laboratory is operated for DOE Office of Science by UChicago Argonne, LLC, under contract number DE-AC02-06CH11357. In addition, we would like to acknowledge the Advanced Cyberinfrastructure for Education and Research (ACER) group at The University of Illinois at Chicago (https://acer.uic.edu), for providing HPC resources that have contributed to the research results reported in this paper.

\section{Author contributions}

Y.Y., J.L., and S.W. designed and directed the study. B.J. and Y.Y. carried out the syntheses of nanocrystals and SERS measurements. Y.F.Y. and Y.Y. carried out TEM and HRTEM characterizations/analyses. W.W. helped with scanning electron microscopy characterizations. C.Z. helped with SERS measurements and related data analysis. W.C helped with the analysis and discussion of TEM results and growth mechanism. V.Y. performed the DFT calculations. K.H. performed TEM characterizations and result in discussions in the second revision. B.J., Y.Y., Y.F.Y., S.W., J.L., and V.Y. wrote the manuscript with contributions from all the authors.

\section{Competing interests}

The authors declare no competing interests.

\section{Additional information}

Supplementary information The online version contains supplementary material available at https://doi.org/10.1038/s41467-021-25969-7.

Correspondence and requests for materials should be addressed to Yun Yang, Shun Wang, Vitaliy Yurkiv or Jun Lu.

Peer review information Nature Communications thanks Zhaochuan Fan and the other, anonymous, reviewer(s) for their contribution to the peer review of this work.

Reprints and permission information is available at http://www.nature.com/reprints

Publisher's note Springer Nature remains neutral with regard to jurisdictional claims in published maps and institutional affiliations.

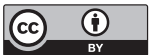

Open Access This article is licensed under a Creative Commons Attribution 4.0 International License, which permits use, sharing, adaptation, distribution and reproduction in any medium or format, as long as you give appropriate credit to the original author(s) and the source, provide a link to the Creative Commons license, and indicate if changes were made. The images or other third party material in this article are included in the article's Creative Commons license, unless indicated otherwise in a credit line to the material. If material is not included in the article's Creative Commons license and your intended use is not permitted by statutory regulation or exceeds the permitted use, you will need to obtain permission directly from the copyright holder. To view a copy of this license, visit http://creativecommons.org/ licenses/by/4.0/.

This is a U.S. Government work and not under copyright protection in the US; foreign copyright protection may apply 2021 